\title{
An Unusual Cause of Small Bowel Obstruction: Appendicular Tourniquet
} M. Ait Chtouk ${ }^{1 *}$, M. Ouali Idrissi ${ }^{1}$, M. Ranib ${ }^{1}$, M. Boukhfaoui ${ }^{2}$, H. Tamour ${ }^{2}$ Louzi $^{2}$, Rabbani ${ }^{2}$, Finech ${ }^{2}$

${ }^{1}$ Radiology Department, Arrazi hospital, CHU Med VI, Marrakesh, Morocco

${ }^{2}$ General surgery department, Arrazi hospital, CHU Med VI, Marrakesh, Morocco

DOI: $10.36347 /$ sjmcr.2021.v09i01.029

| Received: 27.12.2020 | Accepted: 11.01.2021 | Published: 30.01.2021

*Corresponding author: Ait Chtouk Mohamed

\section{Abstract}

Small bowel obstruction is one of the common acute abdomen with different causes. Management of small bowel obstruction needs through knowledge of the pathophysiology and causes of obstruction. The commonest causes include postoperative adhesions, Hernia, volvulus and malignancies. Mechanical small bowel obstruction secondary to acute appendicitis is an uncommon complication of a very common disease. We report an unusual case of an inflamed appendix forming a tourniquet around the terminal ileum producing strangulation.

Keywords: Closed loop obstruction, appendicular tourniquet, laparotomy.

Copyright $(\odot 2021$ The Author(s): This is an open-access article distributed under the terms of the Creative Commons Attribution 4.0 International License (CC BY-NC 4.0) which permits unrestricted use, distribution, and reproduction in any medium for non-commercial use provided the original author and source are credited.

\section{BACKGROUND}

Intestinal obstruction is one of the common surgical emergencies seen in daily practice. Postoperative adhesions are notorious for being the most common cause for intestinal obstruction. Occasionally, laparotomy findings do come as a surprise to surgeons. Here one such case is discussed. A patient was operated on with suspicion of intestinal obstruction secondary to postoperative adhesions. However, laparotomy revealed the appendix to be inflamed, curled around the terminal ileum and acting as a tourniquet.

\section{Case Presentation}

This case report is of 73-year-old male patient with no prior abdominal surgery or comorbidities, who presented to our emergency department with abdominal distension and pain associated with an absolute constipation of aweek duration. On physical examination, he was conscious, alert, hemodynamically stable with no signs of dehydration.

His abdomen was diffusely distended and tenderness, hyper tympanic to percussion and hyperactive bowel sound with no hernias; A digital rectal examination was unremarkable.

Laboratory investigations showed a white cell count of 10,6.103 with neutrophil $80 \%$ whereas biochemistry was normal.
X-ray abdomen reveled dilated small bowl loops with multiples air fluid levels suggestive of intestinal obstruction (Fig-1).

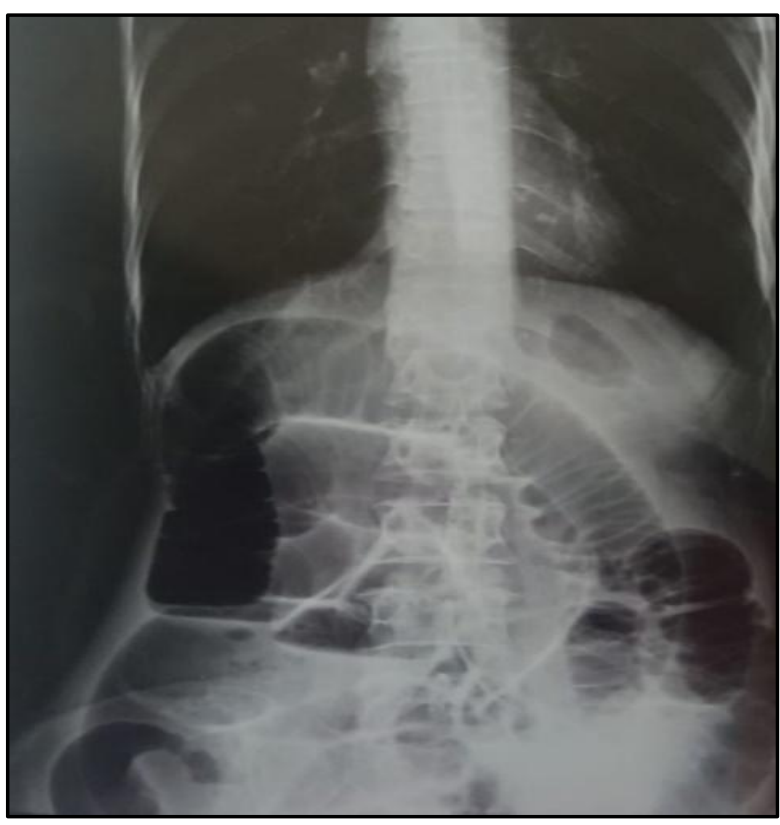

Fig-1: Abdominal X-ray showed multiple air fluid levels central wider than high suggestive of a small bowl obstruction

Computed tomography (CT) with intravenous contrast showed a small bowel obstruction with transition zone in which to points at terminal ileum with bird's beak sign associated to peritoneal effusion suggestive of closed loop obstruction secondary to probable primitive adhesion band (Fig 2, 3, 4 \& 5). 


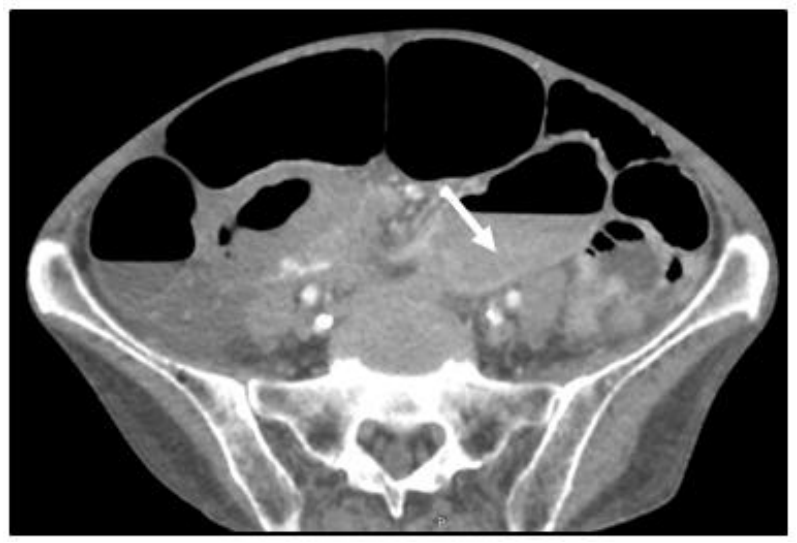

Fig-2: CT with intravenous contrast on axial and coronal slices reveled a transition zone with bird's beak sign (White arrow)

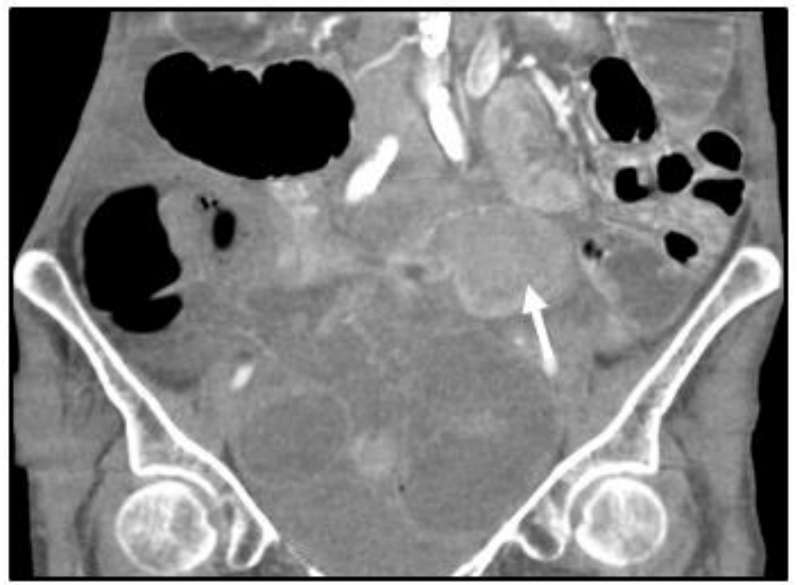

Fig-3: CT with intravenous contrast on axial and coronal slices reveled a transition zone with bird's beak sign (White arrow)

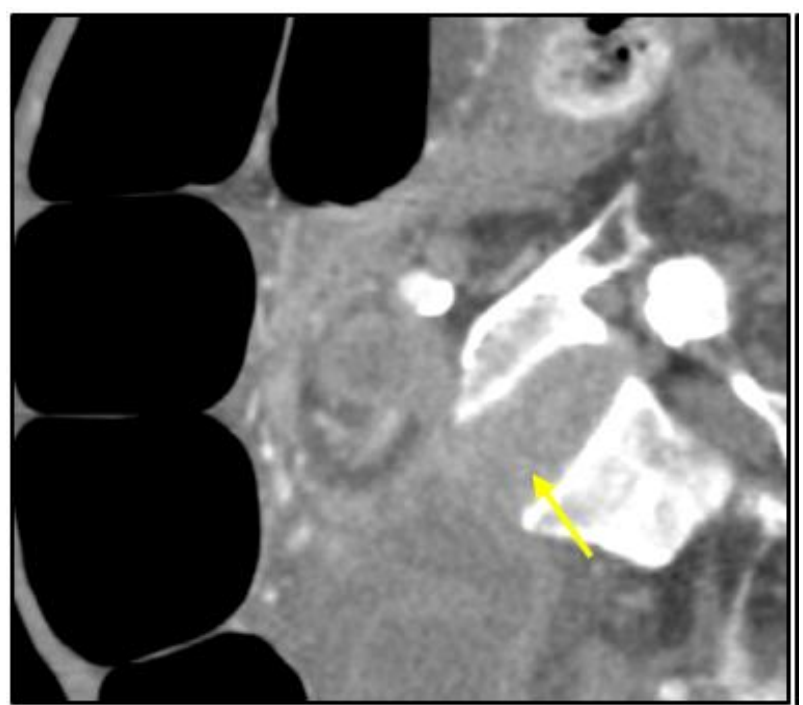

Fig-4: CT with intravenous contrast on sagittal and axial slices reveled appendicular tourniquet (yellow arrow) (retrospectively)

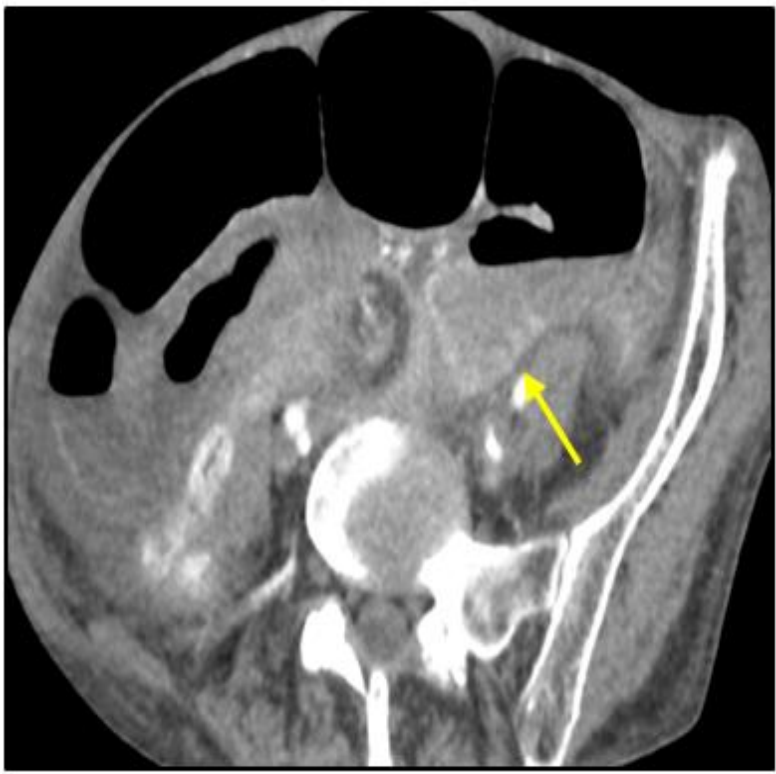

Fig-5: CT with intravenous contrast on sagittal and axial slices reveled appendicular tourniquet (yellow arrow) (retrospectively)

A midline laparotomy was performed under general anesthesia showed dilated small bowel loops to a point where a long-inflamed appendix was wrapped around a loop of terminal ileum at two levels causing closed loop obstruction (Fig 6, 7, 8 \& 9).

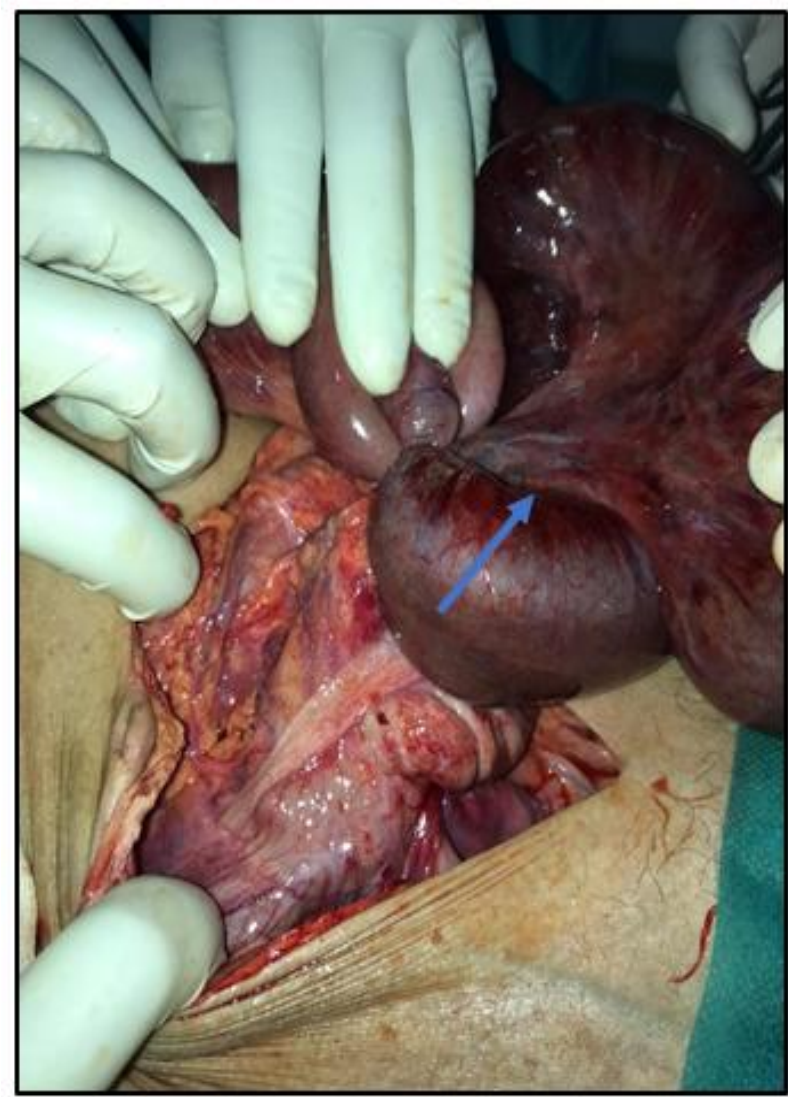

Fig-6: Intraoperative images. Inflamed appendicular tourniquet encircling the terminal ileal loops (blue arrow) 


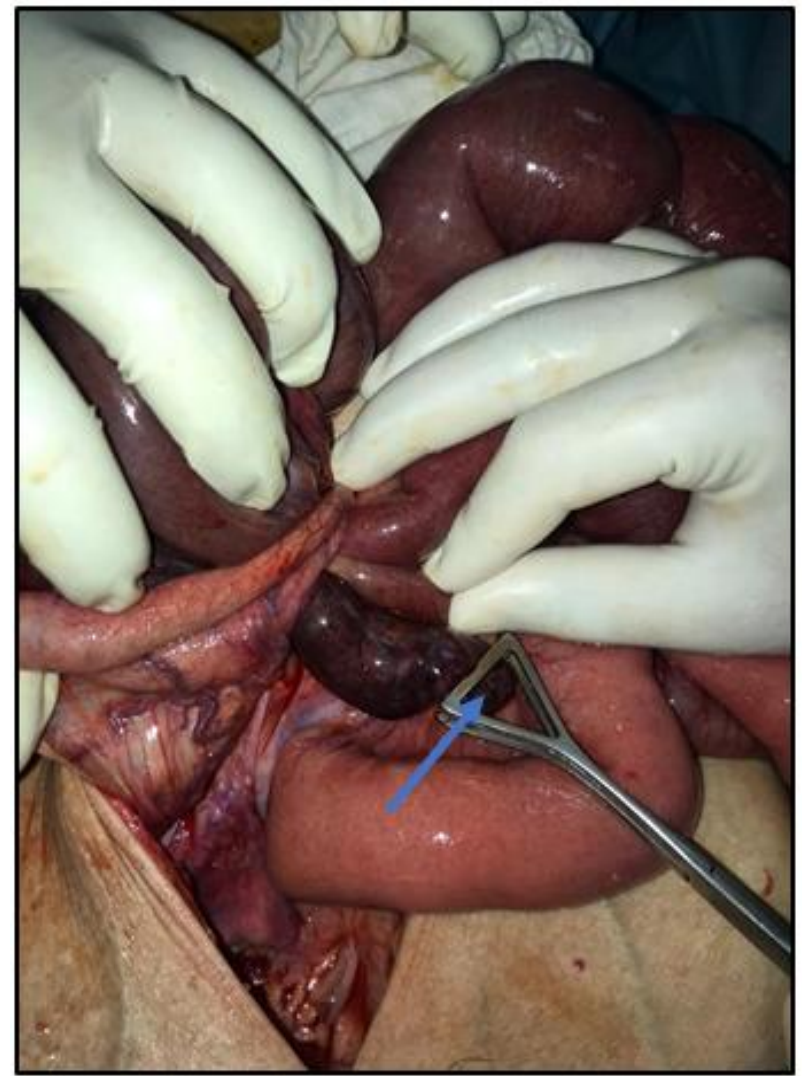

Fig-7: Intraoperative images. Inflamed appendicular tourniquet encircling the terminal ileal loops (blue arrow)

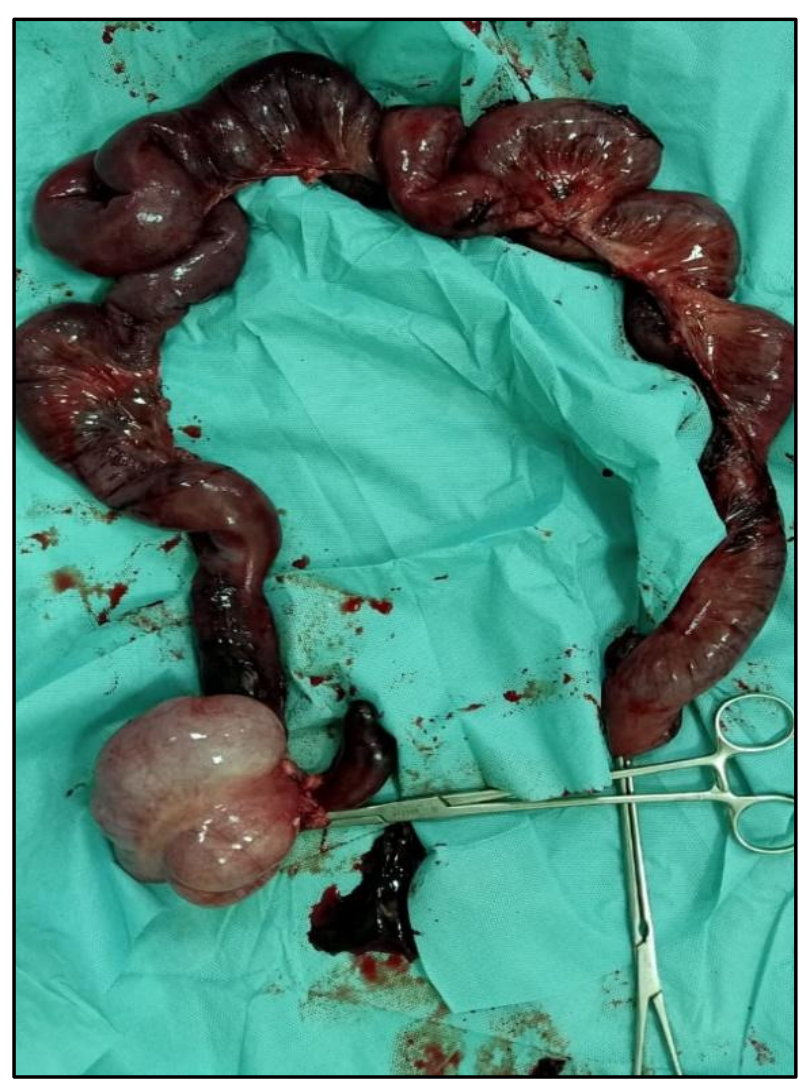

Fig-8: Intraoperative images. Ischemic loop with inflamd appendix

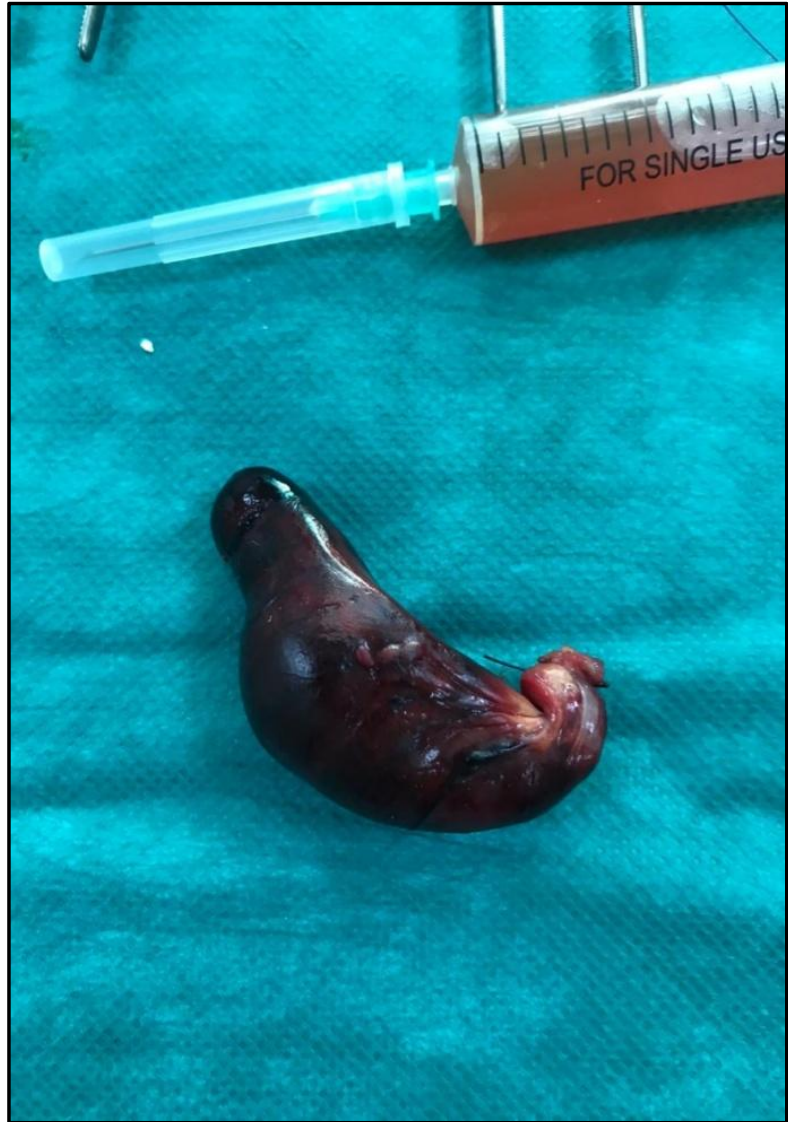

Fig-9: Intraoperative images. Ischemic loop with inflamd appendix

The bowel loops were assessed and found to be necrotic. A wide resection of ischemic loop and appendectomy was done, one tube drain was inserted, and his abdomen was closed in layers.

Histopathological assessment of the appendix revealed features of acute appendicitis.

He was started on orally administered fluids on the third postoperative day and was discharged on the seventh postoperative day.

\section{DISCUSSION}

Acute SBO is one of the most common surgical clinical problems. It can be classified based on many factors: functional or mechanical, the small bowel (SBO) or large bowel (LBO) obstruction, partial or complete, simple, or complicated [1].

Mechanical SBO has many etiologist, post-op adhesion being top in the developed countries. Other common etiologist includes post-op adhesion and small bowel malignancies.

Appendicitis as a cause of $\mathrm{SBO}$ has been known in the literature since 1901 when Lucius Hotchkiss reported three successful surgeries for intestinal obstruction due to appendicitis [2]. 
Bhandari et al., has reviewed the literature and proposed four categories on the pathology of appendicitis causing obstruction; adynamic, mechanical (without strangulation), strangulation of intestine and intestinal obstruction due to mesenteric ischemia [3].

Strangulation can be due to the appendix wrapping around the base of a bowel loop, or when inflamed appendix adheres to caecum, small intestine or posterior peritoneum and a part of the bowel herniates through the gap. This is described with appendicular KNOT, appendicular tourniquet, appendiceal tie syndrome and appendico-ilial knotting [4-7] in our opinion,appendico-ilial knotting is the preferred name because it describes the pathologies of both appendix and ilium.

\section{CONCLUSION}

Intestinal obstruction, although common, can have surprising intraoperative findings. Appendix forming a tourniquet around the terminal ileum, a remote cause of intestinal obstruction qualifies as one such finding. This finding intraoperatively can come as a surprise to a novice surgeon and hence knowledge about such rare causes can help in successful management of patients

\section{REFERENCES}

1. Holzheimer RG, Mannick JA. Surgical Treatment: Evidence Based and Problem-Oriented. Munich: Zuckschwerdt, 2001.

2. Hotchkiss Lucius W. Acute intestinal obstruction following appendicitis. A report of three cases successfully operated upon. Ann Surg. 1901;34:660-77.

3. Mohandas PG, Bhandari L. Appendicitis as a cause of intestinal strangulation: a case report and review. World J Emerg Surg. 2009;4:34.

4. Ranjan A, Kumari K, Jha S. Acute small bowel obstruction as a result of an appendicular knot encircling the terminal ileum:an exceptionally rare case report. Int $\mathbf{J}$ Med Sci Public Health. 2015;4:426-9.

5. Awale L, Joshi BR, Rajbanshi S, Adhikary S. Appendiceal tie syndrome: a very rare complication of a common disease.World $\mathbf{J}$ GastrointestSurg, 27 2015;7:67-70.

6. Yang $\mathrm{AD}$, Lee $\mathrm{CH}$. Appendico-ileal knotting resulting in closed-loop obstruction in a child. PediatrRadiol. 2002;32:879-81.

7. Chowdary PB, Shivashankar SC, Gangapp RB, Varghese EV. Appendicular tourniquet: a cause of intestinal obstruction. J ClinDiagn Res. 2016;10:9-11. 